\title{
Prostate-specific antigen density as the best predictor of low- to intermediate-risk prostate cancer: A cohort study
}

Dae Hyoung Park

INJE University Sanggye Paik Hospital

Jae-Yoon Kim

INJE University Sanggye Paik Hospital

Dae Yeon Cho

INJE University Sanggye Paik Hospital

Luck Hee Sung

INJE University Sanggye Paik Hospital

Ji Hyeong Yu ( $\square$ uroone@paik.ac.kr)

INJE University Sanggye Paik Hospital

\section{Research Article}

Keywords: prostate-specific antigen density, prostate cancer, lower urinary tract symptoms, digital rectal examination

Posted Date: February 4th, 2022

DOI: https://doi.org/10.21203/rs.3.rs-1298769/v1

License: (9) (1) This work is licensed under a Creative Commons Attribution 4.0 International License. Read Full License 


\section{Abstract}

Background: Evaluation of prostate cancer ( $\mathrm{PCa}$ ) when serum prostate-specific antigen (PSA) level is vaguely elevated is complicated. This is because serum PSA levels only reflect the number of prostate epithelial cells. We aimed to compare PSA and various prostate volume-related factors to determine which one can best predict PCa in patient with a PSA level of $2.5-20 \mathrm{ng} / \mathrm{mL}$.

Methods: Patients who underwent transrectal ultrasound (TRUS)-guided prostate biopsy at the Inje University Sanggye Paik Hospital between January 2018 and July 2021 and who had a PSA level of 2.5$20 \mathrm{ng} / \mathrm{mL}$ were retrospectively identified $(\mathrm{n}=275)$. Among them, based on biopsy results, patients were divided into cancer group and non-cancer groups, and age, PSA, total prostate volume (TPV), peripheral zone volume (PZV), peripheral zone PSA density (PZ-PSAD), transitional zone-PSAD (TZ-PSAD), and PSAD were compared and analyzed using receiver operating characteristic (ROC) and univariate analyses.

Results: The areas under ROC curves (AUCs) for age, total PSA, TPV, PZV, PZ-PSAD, TZ-PSAD, and PSAD for predicting PCa in patients with a PSA level of $2.5-20.0 \mathrm{ng} / \mathrm{mL}$ were $0.678,0.680,0.671,0.639,0.731$, 0.736 , and 0.764 , respectively. In univariate analysis, all categorical variables were divided based on the cut-off value and used to predict PCa. Those with a PSAD of $\geq 0.218 \mathrm{ng} / \mathrm{mL}^{2}$ were found to be at an increased risk of PCa than those with a PSAD of $<0.218 \mathrm{ng} / \mathrm{mL}^{2}$ (odds ratio [OR] 8.16, 95\% confidence interval [CI] 4.612-14.419), which was the best result, followed by TZ-PSAD with a cut-off value of 0.353 , PZ-PSAD of 0.368 , TPV of 34.50 , PSA of 6.315 , age of 68.5 , and PZV of $19.85 \mathrm{~mL}$. At a PSAD level of $0.218 \mathrm{ng} / \mathrm{mL}^{2}, 85.0 \%$ of the PCa group could avoid unnecessary biopsy and $61.4 \%$ of the non-PCa group could reduce missed diagnosis when the TRUS findings were inaccurate.

Conclusions: PSAD may inform biopsy decisions as the best predictor of PCa when TRUS findings are ambiguous in patients with a PSA level of $2.5-20.0 \mathrm{ng} / \mathrm{mL}$.

\section{Background}

Prostate cancer (PCa) is the second most commonly diagnosed cancer in men worldwide, and its global incidence in 2018 was 29.3 per 100,000 individuals [1]. PCa is also the fourth leading cause of cancer death in men, with a mortality rate of 7.6 per 100,000 individuals [1].

Lower urinary tract symptoms (LUTS) are one of the main problems that adversely affect quality of life among elderly individuals. The prevalence of LUTS increases from $44 \%$ in men aged $40-59$ years to $70 \%$ in men aged over 80 years [2]. Benign prostatic hyperplasia (BPH) is the most common cause of LUTS, although neurological factors and infections may also cause LUTS [3]. Because prostate-specific antigen (PSA) level increases in PCa as well as in $\mathrm{BPH}$ or other conditions including prostatitis, it is important that $\mathrm{BPH}$ and $\mathrm{PCa}$ are not misdiagnosed to guide appropriate treatment. 
Currently, cancer evaluation is routinely performed when patients have a PSA level of $>2.5 \mathrm{ng} / \mathrm{mL}$ or if any abnormality is noted on digital rectal examinations (DREs). However, several men undergo unnecessary prostate biopsy when only PSA level is used for screening [4]. Although many studies have attempted to utilize PSA as a means of PCa diagnosis, they have not been successful because of the lack of specificity of PSA for PCa or BPH [5]. Therefore, identifying individuals who need to undergo biopsy, and thereby avoiding unnecessary procedures to minimize complications such as pain, infection, bleeding, and sepsis, is vital.

Besides PSA, many factors, including total prostate volume (TPV), PSA density (PSAD), ratio of free PSA to PSA, and transition zone index (TZl; ratio of the transition zone volume [TZV] to the TPV), have been studied as predictive factors for PCa. However, most studies have assessed these predictors in patients at low risk of PCa [6-10]. Although the Prostate Imaging-Reporting and Data System (PI-RADS) score of multiparameter magnetic resonance imaging (mpMRI) can increase the diagnosis rate, it still has limitations, such as high cost and long waiting time. Therefore, this study aimed to examine best predictors of PCa among patients with PSA levels of $2.5-20.0 \mathrm{ng} / \mathrm{mL}$ who have a low-to-intermediate risk of PCa and to investigate associations between PCa and age, total PSA, TPV, PZV, peripheral zone-PSAD (PZ-PSAD), transitional zone-PSAD (TZ-PSAD), and PSAD.

\section{Methods}

\section{Study design}

After obtaining approval from the Institutional Review Board (IRB) of the Inje University, we evaluated the records of 512 patients who underwent transrectal ultrasound prostate biopsy (TRUS-Bx) at our clinic between January 2018 and July 2021 and who had a high PSA level or abnormal findings in TRUS or DRE. The study protocol was reviewed by the IRB of Inje University of Sanggye Paik Hospital and they waived the need for informed consent in the institutions.

Patients were excluded if they had a PSA level below $2.5 \mathrm{ng} / \mathrm{mL}$ or above $20 \mathrm{ng} / \mathrm{mL}$, received 5-alphareductase inhibitors over 3 months in the previous 2 years, or were diagnosed with atypical small acinar proliferation (ASAP) or high-grade prostate intraepithelial neoplasia. Other exclusion criteria were a history of other prostate surgeries including prostate biopsy, prostatitis, urinary retention within 1 year, or missed examination. After applying the exclusion criteria, a total of 275 patients were included in the study (Figure 1).

In patients with suspected PCa, PSA levels were measured in $\mathrm{ng} / \mathrm{mL}$ by immunochemical analysis before biopsy, followed by DRE and TRUS. PSA levels were determined using a Cobas 8000 modular analyzer series (Roche Diagnostics International Ltd., Basel, Switzerland).

\section{Study protocol}


Five urologists (LH, JH, DY, JY, and HD) with more than 5 years of experience performed TRUS-Bx. Patients lay on the left side with both knees bent. Local anesthesia with $1 \%$ lidocaine was administered, and a urologist used a spring-loaded needle to collect 12-15 tissue samples from different parts of the gland. TRUS was performed to compute TPV and TZV by measuring the length, height, and width of the gland and multiplying the product by a coefficient of $\pi / 6$ (ellipsoid formula). PZV was calculated as the difference between the TPV and TZV. PZ-PSAD and TZ-PSAD were measured by dividing the PSA level by PZV and TZV, respectively. PSAD was obtained by dividing the PSA level by TPV. Based on biopsy results, patients were divided into a cancer group and non-cancer group, and basic characteristics such as age, total PSA, TPV, PZV, PZ-PSAD, TZ-PSAD, PSAD, and Gleason score were collected for each group. Clinically significant PCa (csPCa) was defined as a Gleason score of $\geq 7$ or Gleason score of 6 , with $\geq 3$ positive and/or maximum core participation of $\geq 50 \%$. ( $(\mathbb{Q} \mathbb{1} \mathrm{Q} 13$ )

\section{Statistical analysis}

Statistical comparisons of the cancer and non-cancer groups were performed using the independent t-test or Kolmogorov-Smirnov (K-S) test for parametric or nonparametric analysis, respectively. Receiver operating characteristic (ROC) curve analyses were performed to determine optimal cutoffs for age, total PSA, TPV, PZV, PZ-PSAD, TZ-PSAD, and PSAD for PCa diagnosis. Continuous data were compared using the t-test and K-S test, and categorical data were compared using the Pearson chi-square test, Fisher's exact test, and chi-square test. Univariate logistic regression analyses were performed to compare age, total PSA, TPV, PZV, PZ-PSAD, TZ-PSAD, and PSAD in patients with a PSA level of $2.5 .0-20.0 \mathrm{ng} / \mathrm{mL}$ to predict PCa. All analyses were performed using SPSS (version 22.0; IBM, New York, NY, USA). Comparisons were two sided, and differences were considered statistically significant at $p$-values of $<0.05$.

\section{Results}

There were significant differences between the PCa and non-PCa groups with respect to age, total PSA, TPV, PZV, PZ-PSAD, TZ-PSAD, and PSAD (all $p<0.001$ ). Age, PSA, PZ-PSAD, TZ-PSAD, and PSAD were higher in the PCa group than in the non-PCa. group. TPV and PZV were significantly lower in the PCa group than in the non-PCa. group. The presence of an abnormal DRE finding was higher in the PCa group than in the non-PCa group $(p<0.05)$. The presence of an abnormal finding in TRUS, however, showed no significant difference between the two groups. In the PCa group, the number of patients with clinically significant cancer was 99 (89.2\%). The non-PCa group had more chronic inflammation than the PCa group $(n=124,75.6 \%$ vs $n=52,46.8 \%)$ (Table 1$)$.

Chronic inflammation was detected in 124 patients (75.6\%) in the non-PCa group and in 52 patients in the PCa group (46.8\%). Patients with PCa were older and had higher values of PZ-PSAD, PSA, PSAD, and TZ-PSAD and positive findings on DRE and TRUS than patients with benign pathology (Table 1).

Table 1. Comparison of characteristics between the cancer and non-cancer groups 


\begin{tabular}{|llll|}
\hline & $\begin{array}{l}\text { Non-PCa group } \\
(\mathrm{n}=164)\end{array}$ & $\begin{array}{l}\text { PCa group } \\
(\mathrm{n}=111)\end{array}$ & $\mathrm{p}$-value \\
\hline Age (years) Mean \pm SD & $66.9 \pm 7.98$ & $72.1 \pm 7.3$ & $<0.001^{\mathrm{a}}$ \\
\hline Total PSA $(\mathrm{ng} / \mathrm{mL})$ Mean $\pm \mathrm{SD}$ & $6.86 \pm 3.07$ & $9.01 \pm 3.77$ & $<0.001^{\mathrm{a}}$ \\
\hline TPV $(\mathrm{mL})$ Mean \pm SD & $52.22 \pm 26.54$ & $39.69 \pm 18.13$ & $<0.001^{\mathrm{a}}$ \\
\hline $\mathrm{PZV}(\mathrm{mL})$ Mean \pm SD & $25.32 \pm 11.69$ & $20.03 \pm 8.35$ & $<0.001^{\mathrm{a}}$ \\
\hline PZ-PSAD $\left(\mathrm{ng} / \mathrm{mL}^{2}\right)$ Mean $\pm \mathrm{SD}$ & $0.319 \pm 0.198$ & $0.536 \pm 0.336$ & $<0.001^{\mathrm{a}}$ \\
\hline TZ-PSAD $\left(\mathrm{ng} / \mathrm{mL}^{2}\right)$ Mean $\pm \mathrm{SD}$ & $0.362 \pm 0.360$ & $0.644 \pm 0.433$ & $<0.001^{\mathrm{a}}$ \\
\hline PSAD (ng/mL $\left.{ }^{2}\right)$ Mean $\pm \mathrm{SD}$ & $0.154 \pm 0.950$ & $0.270 \pm 0.160$ & $<0.001^{\mathrm{a}}$ \\
\hline Abnormal finding in DRE, $\mathrm{n}(\%)$ & $6(3.7)$ & $11(9.9)$ & $0.035^{\mathrm{b}}$ \\
\hline Abnormal finding in TRUS, $\mathrm{n}(\%)$ & $60(36.6)$ & $41(36.9)$ & $0.953^{\mathrm{b}}$ \\
\hline Clinically significant cancer, $\mathrm{n}(\%)$ & - & $99(89.2)$ & \\
\hline Chronic inflammation, $\mathrm{n}(\%)$ & $124(75.6)$ & $52(46.8)$ & $<0.001^{\mathrm{b}}$ \\
\hline
\end{tabular}

PCa: prostate cancer; PSA: prostate-specific antigen; TPV: total prostate volume; PZV: peripheral zone volume; PZ-PSAD: peripheral zone prostate-specific antigen density; TZ-PSAD: transition zone prostatespecific antigen density; DRE: digital rectal examination; TRUS: transrectal ultrasound; SD: standard deviation.

andependent t-test

${ }^{b}$ Pearson's chi-squared test

The areas under the ROC curves (AUCs) for age, total PSA, TPV, PZV, PZ-PSAD, TZ-PSAD, and PSAD for predicting PCa in patients with a PSA level of $2.5-20.0 \mathrm{ng} / \mathrm{mL}$ were $0.678,0.680,0.671,0.639,0.731$, 0.736 , and 0.764 , respectively (Table 2). PSAD was a significantly better predictor of PCa in this group than age, total PSA, TPV, PZV, PZ-PSAD, or TZ-PSAD. TZ-PSAD was the next best predictor, followed by PZ-PSAD, total PSA, age, TPV, and PZV (Table 2 and Figure 2). The ROC curve analysis revealed the optimal cut-off value for PSAD to be 0.218 in patients with a PSA level of $4.0-20.0 \mathrm{ng} / \mathrm{mL}$. At a cut-off value of 0.218 , sensitivity and specificity were $59.5 \%$ and $84.8 \%$, respectively.

For predicting csPCa in patients, the AUCs for age, total PSA, TPV, PZV, PZ-PSAD, TZ-PSAD, and PSAD were $0.687,0.659,0.662,0.632,0.714,0.717$, and 0.745 , respectively (Table 3 ). PSAD was significantly the best predictor of csPCa in this group. ROC curve analysis revealed an optimal cut-off value of 0.218 
(Figure 2). At this cut-off value, sensitivity and specificity were $59.6 \%$ and $81.8 \%$, respectively. Therefore, PSAD did not improve the detection of csPCa compared with PCa in our clinic.

Table 2. AUC, sensitivity, specificity, and best cut-off at predicting PCa

\begin{tabular}{|lllll|}
\hline & AUC & Sensitivity (\%) & Specificity (\%) & Best cut-off \\
\hline Age & 0.678 & 0.694 & 0.585 & $\geq 68.5$ \\
\hline PSA & 0.680 & 0.730 & 0.555 & $\geq 6.425$ \\
\hline TPV & 0.671 & 0.495 & 0.787 & $<0.029$ \\
\hline PZV & 0.639 & 0.559 & 0.646 & $<0.050$ \\
\hline PZ-PSAD & 0.731 & 0.649 & 0.732 & $\geq 0.368$ \\
\hline TZ-PSAD & 0.736 & 0.712 & 0.689 & $\geq 0.353$ \\
\hline PSAD & 0.764 & 0.595 & 0.848 & $\geq 0.218$ \\
\hline
\end{tabular}

AUC: area under the receiver operating characteristic curve; PSA: prostate-specific antigen; TPV: total prostate volume; PZV: peripheral zone volume; PZ-PSAD: peripheral zone prostate-specific antigen density; TZ-PSAD: transition zone prostate-specific antigen density

Table 3. AUC, sensitivity, specificity, and best cut-off at predicting csPCa

\begin{tabular}{|lllll|}
\hline & AUC & Sensitivity (\%) & Specificity (\%) & Best cut-off \\
\hline Age & 0.687 & 0.616 & 0.676 & $\geq 70.5$ \\
\hline PSA & 0.659 & 0.747 & 0.517 & $\geq 6.315$ \\
\hline TPV & 0.662 & 0.848 & 0.403 & $<0.019$ \\
\hline PZV & 0.632 & 0.727 & 0.466 & $<0.043$ \\
\hline PZ-PSAD & 0.714 & 0.646 & 0.705 & $\geq 0.368$ \\
\hline TZ-PSAD & 0.717 & 0.717 & 0.665 & $\geq 0.353$ \\
\hline PSAD & 0.745 & 0.596 & 0.818 & $\geq 0.218$ \\
\hline
\end{tabular}

AUC: area under the receiver operating characteristic curve; csPCa: clinically significant prostate cancer; PSA: prostate-specific antigen; TPV: total prostate volume; PZV: peripheral zone volume; PZ-PSAD: peripheral zone prostate-specific antigen density; TZ-PSAD: transition zone prostate-specific antigen density

In univariate analysis, all categorical variables were divided based on the cut-off value and used to predict PCa. Among patients with a PSA level of $2.5-20.0 \mathrm{ng} / \mathrm{mL}$, those with a PSAD of $\geq 0.218 \mathrm{ng} / \mathrm{mL}^{2}$ 
were found to be at an increased risk of PCa than those with a PSAD of $<0.218 \mathrm{ng} / \mathrm{mL}^{2}$ (odds ratio [OR] 8.16, 95\% confidence interval [CI] 4.612-14.419), which was the best result, followed by TZ-PSAD with a cut-off value of 0.353 (OR 5.47, 95\% Cl 3.229-9.268), PZ-PSAD with 0.368 (OR 5.04, 95\% Cl 2.9918.475), TPV with 34.50 (OR 3.62, 95\% Cl 2.137-6.133), PSA with 6.315 (OR 3.62, 95\% Cl 2.137-6.133), age with 68.5 (OR 3.20, 95\% Cl 1.921-5.321), and PZV with 19.85 (OR 2.31, 95\% Cl 1.413-3.785) (Tables 4).

Table 4. Univariate analyses of PCa-associated factors in patients with PSA levels of $2.5-20.0 \mathrm{ng} / \mathrm{mL}$ 


\begin{tabular}{|c|c|c|c|c|}
\hline & \multicolumn{2}{|c|}{ Pathologic report of prostate biopsy } & \multirow[t]{3}{*}{ OR $(95 \% \mathrm{Cl})$} & \multirow[t]{3}{*}{$P$-value } \\
\hline & Non-cancer group & Cancer group & & \\
\hline & $(n=164)$ & $(n=111)$ & & \\
\hline Age (years) & & & $3.20(1.921-5.321)$ & $<0.001$ \\
\hline$<68.5$ & 96 & $34(30.6 \%)$ & & \\
\hline$\geq 68.5$ & 68 & $77(69.4 \%)$ & & \\
\hline PSA (ng/mL) & & & $3.43(2.026-5.814)$ & $<0.001$ \\
\hline$<6.315$ & 88 & 28 & & \\
\hline$\geq 0.315$ & 76 & 83 & & \\
\hline TPV $(\mathrm{mL})$ & & & $3.62(2.137-6.133)$ & $<0.001$ \\
\hline$\geq 4.50$ & 129 & 56 & & \\
\hline$<34.50$ & 35 & 55 & & \\
\hline PZV (mL) & & & $2.31(1.413-3.785)$ & 0.001 \\
\hline$\geq 19.85$ & 106 & 49 & & \\
\hline$<19.85$ & 58 & 62 & & \\
\hline PZ-PSAD (ng/mL²) & & & $5.04(2.991-8.475)$ & $<0.001$ \\
\hline$<0.368$ & 120 & 39 & & \\
\hline$\geq 0.368$ & 44 & 72 & & \\
\hline TZ-PSAD (ng/mL $\left.{ }^{2}\right)$ & & & $5.47(3.229-9.268)$ & $<0.001$ \\
\hline$<0.353$ & 113 & 32 & & \\
\hline$\geq 0.353$ & 51 & 79 & & \\
\hline PSAD (ng/mL $\left.{ }^{2}\right)$ & & & $8.16(4.612-14.419)$ & $<0.001$ \\
\hline$<0.218$ & 139 & 45 & & \\
\hline$\geq 0.218$ & 25 & 66 & & \\
\hline
\end{tabular}

PSA: prostate-specific antigen; TPV: total prostate volume; PZV: peripheral zone volume; PZ-PSAD: peripheral zone prostate-specific antigen density; TZ-PSAD: transition zone prostate-specific antigen density; OR: odds ratio; Cl: confidence interval

We evaluated the efficacy of PSAD when TRUS findings were inaccurate (Figure 3). 
Among 275 patients with PSA levels of $2.5-20.0 \mathrm{ng} / \mathrm{mL}, 60$ patients in the non-PCa group had inaccurate TRUS findings, of whom 51 (85.0\%) patients with PSAD of $<0.218 \mathrm{ng} / \mathrm{mL}^{2}$ could avoid unnecessary biopsies. Additionally, 70 patients in the PCa group had inaccurate TRUS findings; among them, 43 (61.4\%) patients with a PSAD of $\geq 0.218 \mathrm{ng} / \mathrm{mL}^{2}$ could avoid missed diagnosis.

\section{Discussion}

To the best of our knowledge, this is the first study to investigate PSAD as a risk factor for PCa in patients with low-to-intermediate risk (PSA levels $2.5-20.0 \mathrm{ng} / \mathrm{mL}$ ). We found that the risk of PCa increased in this patient cohort when PSAD was 0.218 or higher.

In patients with high PSA levels, PCa should be excluded based on TRUS-Bx findings. For patients with enlarged prostates, assuming there is no acute inflammation or urinary retention, biopsy can be used to confirm the lack of cancer, which would indicate that the increase in PSA levels is because of enlarged prostate or chronic inflammation. However, TRUS-Bx may be unnecessary in such cases. In this study, $164(59.6 \%)$ of 275 patients with a PSA level of $2.5-20.0 \mathrm{ng} / \mathrm{mL}$ were not diagnosed with PCa, indicating they had elevated PSA levels caused by BPH or chronic inflammation. Therefore, it is important to identify patients who would require biopsy to minimize complications such as bleeding, infection, and pain and avoid unnecessary procedures.

We conducted this retrospective study of patients with no evidence of acute inflammation and a PSA level of $2.5-20.0 \mathrm{ng} / \mathrm{mL}$. It is not easy to identify which patients in this cohort have PCa. Porcaro et al. showed that a higher prostate volume index, defined as the ratio of TZV to PZV, and the presence of prostatic chronic inflammation predicted a decreased risk of $\mathrm{PCa}$ in patients with normal DRE findings and a PSA level of $2.0-10.0 \mathrm{ng} / \mathrm{mL}$ [6]. Kalish et al. reported that serum PSA levels adjusted for TZV were more accurate in predicting cancer than PSA alone among patients with PSA levels of $4.0-10.0 \mathrm{ng} / \mathrm{mL}$ [11]. Roobol et al. suggested that PCa screening could be improved by a calculation method using prostrate volume (PV) data from TRUS at the European Randomized Study of Screening for Prostate Cancer [4]. Additionally, Freedland et al. showed that a lower PV was associated with more advanced cancer and high-grade disease among patients with PCa [12]. In all of these studies, the authors indicate that PSA levels should not be used alone, but rather we should refer to prostate volume for a more accurate diagnosis of PCa.

A risk assessment based solely on PSA is not optimal. PSAD has been proposed to predict biochemical recurrence after prostatectomy better than PSA [13]. Therefore, when PSA is ambiguously elevated (PSA levels $2.5-20.0 \mathrm{ng} / \mathrm{mL}$ ), it can be further refined by assessing PSAD. Our study showed that PSAD was most associated with PCa risk in patients with a PSA level of $2.5-20.0 \mathrm{ng} / \mathrm{mL}$. We suggest that patients with a PSA level of $2.5-20.0 \mathrm{ng} / \mathrm{mL}$ should be carefully examined for malignancy and that PSAD should be considered before performing mpMRI or biopsy. 
Although there is no clear molecular or genetic relationship between $\mathrm{BPH}$ and $\mathrm{PCa}$, suggesting two distinct etiological pathways, epidemiologic studies have shown that both conditions are hormone dependent and associated with prostate inflammation, which can represent a common denominator, elevated PSA levels [14]. In this study, 176 of 275 patients with a PSA level of $2.5-20.0 \mathrm{ng} / \mathrm{mL}$ had chronic inflammation. Microarray studies revealed overlap in gene clusters associated with inflammation between $\mathrm{BPH}$ and PCa [15]. Obesity, diabetes, dyslipidemia, and high blood pressure have all been identified as risk factors for the development of BPH and PCa [16-18]. The studies argue that these two diseases should be regarded as metabolic syndromes, which are related to chronic inflammation, because they do not have separate pathologies and are associated with complex factors such as geriatric disease.

Many studies have shown that PSAD is more effective than PSA in predicting PCa [19-21]. Although they only mentioned the gray zone (PSA level upper normal limit $10 \mathrm{ng} / \mathrm{mL}$ ), our results suggest that a PSA level up to $20 \mathrm{ng} / \mathrm{mL}$ could also help predict cancer based on PSAD. Verma et al. showed that the PSADs of 141 patients with PSA 10 or higher had higher AUC values than those of PSA within the gray zone (0.72 vs 0.61 ), showing better results in predicting PCa [22]. Therefore, extending the PSA range, as reflected in our study, does not appear to significantly change the prediction of $\mathrm{PCa}$.

Kalish et al., assuming that BPH is mainly induced in TZ and that PSA change due to BPH is caused by an enlarged gland in TZ, showed that TZ-PSAD calculated by adjusting PSA with TZV is the most important predictor in predicting PCa [11]. However, on the contrary, Wang et al. reported that PZ-PSAD was the most important in predicting PCa by increasing the positivity rate of biopsy from $21.7 \%$ to $54.7 \%$ when TRUS and mpMRI were ambiguous [23]. There are also several studies that state that TZ-PSAD is not superior to PSAD in PCa prediction $[20,24]$.

Our study showed that PSAD performed better than PZ-PSAD, TZ-PSAD, and PSA in univariate analyses of PCa-associated factors. Patients with a PSAD of $0.218 \mathrm{ng} / \mathrm{mL}^{2}$ or greater had an eight-fold increased risk of PCa compared with patients with a PSAD of $<0.218 \mathrm{ng} / \mathrm{mL}^{2}$.

Lee et al. reported that it was most helpful in predicting PCa at a volume-adjusted PSAD of $0.18 \mathrm{ng} / \mathrm{mL}^{2}$ or higher (OR 5.171, 95\% $\mathrm{Cl} 3.171-8.432, \mathrm{P}<0.001)$. They reported an increase in cancer detection when raising the PSAD cut-off value from 0.15 to 0.18 (positive predictive value $70.3 \%$, negative predictive value 68.6\%) [21]. Moreover, Liu et al. proposed the best cut-off value of PSAD in the predictive model for the csPCa group to be 0.20 [25]. In our study, when csPCa was compared with PCa, the AUC was not significantly higher, but the same cut-off value was presented. By suggesting the higher value of 0.218 in our current study, we might compensate for the masking of cancer detection by the large PV.

Prostate Health Index $(\mathrm{PHI})$ is a novel screening tool presented in a prospective multi-center studies that can improve the prediction of PCa along with the 4K score and is suggested in the current guidelines $[26,27]$. It is calculated using the following formula: (p2PSA / free PSA) $\times \sqrt{ }$ total PSA. Chiu et al. reported that $\mathrm{PHI}$ density obtained by dividing PHI by PV value was an excellent predictor of csPCa, and at $90 \%$ 
sensitivity, reduced unnecessary biopsies (43.7\%) and missed the fewest csPCa (8.5\%) [28]. Although our clinic has not been able to perform the PHI test in the absence of p2PSA to date, there seems to be no disagreement on the premise that cancer detection should be improved by referring to the prostate volume. In addition, since the half-life of free PSA is approximately $2 \mathrm{~h}$ [29], it is necessary to refer to it in consideration of laboratory conditions. As shown in Figure 3, our study showed that at PSAD of 0.218 , $85.0 \%$ of the PCa group could avoid unnecessary biopsy and $61.4 \%$ of the non-PCa group could reduce avoid diagnosis when the TRUS findings were inaccurate.

Our study has some limitations. First, it was retrospective and conducted at a single institution. Second, we measured TPV using TRUS rather than prostatectomy specimens, and using the ellipsoid formula underestimates PV by $18 \%$ [30]. However, it would have been impossible to confirm tumor volume through prostatectomy in all patients with PCa because watchful waiting, active surveillance, hormone therapy, and radiation therapy were performed in addition to surgery. Third, variability among different operators was not considered. Lastly, some cancers may have been undiagnosed.

However, our findings are expected to be helpful in screening patients for PCa. Alongside PSA, the cancer detection rate could be increased when PSAD was 0.218 or higher in patients with PSA levels of 2.5-20.0 $\mathrm{ng} / \mathrm{mL}$.

\section{Conclusions}

Various models have been proposed to predict the diagnosis of PCa, but none have yet been definitive, and our study suggests that PSAD is the best model for PSA levels of $2.5-20 \mathrm{ng} / \mathrm{mL}$.

PSAD may inform biopsy decisions as the best predictor of PCa when TRUS findings were ambiguous in patients with a PSA level of $2.5-20.0 \mathrm{ng} / \mathrm{mL}$.

\section{Abbreviations}

AUC: area under the curve

$\mathrm{BPH}$ : benign prostatic hyperplasia

DRE: digital rectal examination

GGS: group grade score

K-S: Kolmogorov-Smirnov

LUTS: lower urinary tract symptoms

MRI: magnetic resonance imaging

PCa: prostate cancer

Page $11 / 18$ 
PSA: prostate-specific antigen

PV: prostrate volume

ROC: receiver operating characteristic

TPV: total prostate volume

TRUS-Bx: transrectal ultrasound prostate biopsy

TZI: transition zone index

TZV: transition zone value

\section{Declarations}

\section{Ethics approval and consent to participate}

The study was performed in accordance with the Declaration of Helsinki, and ethics approval was obtained from Ethics Committee of Inje University of Sanggye Paik Hospital (IRB: 2021-10-001). Because of the retrospective nature of this study, the need to obtain informed consent was waived by Ethics Committee of Inje University of Sanggye Paik Hospital.

\section{Consent for publication}

Not applicable.

\section{Availability of data and materials}

The dataset generated and/or analyzed during the current study are not publicly available due to identifable patient information but are available from the corresponding author upon reasonable request.

\section{Competing interests}

The authors declare no potential conflicts of interest

\section{Funding}

The research received no specific grant from any funding agency in the public, commercial, or not-forprofit sectors.

\section{Authors' contributions}

DH was the lead author and conducted the literature review, authored the text, and developed the hypothesis. JY and LH conducted the data analysis, interpretation, and statistical calculations, and DY 
and $\mathrm{JH}$ collaborated on the methods and discussion sections. DH was instrumental in hypothesis development, literature review, and the authoring of the text.

\section{Acknowledgements}

Not applicable.

\section{Authors' information (optional)}

${ }^{1}$ Department of Urology, INJE University Sanggye Paik Hospital, Seoul, Republic of Korea

\section{References}

1. Bray F, Ferlay J, Soerjomataram I, Siegel RL, Torre LA, Jemal A. Global cancer statistics 2018: GLOBOCAN estimates of incidence and mortality worldwide for 36 cancers in 185 countries. CA Cancer J Clin. 2018;68:394-424.

2. Irwin DE, Milsom I, Hunskaar S, Reilly K, Kopp Z, Herschorn S, et al. Population-based survey of urinary incontinence, overactive bladder, and other lower urinary tract symptoms in five countries: results of the EPIC study. Eur Urol. 2006;50:1306-14; discussion 1314.

3. Lee CL, Kuo HC. Videourodynamic analysis in men with lower urinary tract symptoms: correlation between age and prostate size with lower urinary tract dysfunction. Urol Sci. 2016;27:21-5.

4. Roobol MJ, van Vugt HA, Loeb S, Zhu X, Bul M, Bangma CH, et al. Prediction of prostate cancer risk: the role of prostate volume and digital rectal examination in the ERSPC risk calculators. Eur Urol. 2012;61:577-83.

5. Benson MC, Whang IS, Pantuck A, Ring K, Kaplan SA, Olsson CA, et al. Prostate specific antigen density: a means of distinguishing benign prostatic hypertrophy and prostate cancer. J Urol. 1992;147:815-6.

6. Porcaro AB, Tafuri A, Sebben M, Shakir A, Novella G, Pirozzi M, et al. Prostate volume index and prostatic chronic inflammation have an effect on tumor load at baseline random biopsies in patients with normal DRE and PSA values less than $10 \mathrm{ng} / \mathrm{ml}$ : results of 564 consecutive cases. Ther Adv Urol. 2019;11:1756287219868604.

7. Nordström T, Akre O, Aly M, Grönberg H, Eklund M. Prostate-specific antigen (PSA) density in the diagnostic algorithm of prostate cancer. Prostate Cancer Prostatic Dis. 2018;21:57-63.

8. Erdogan A, Polat S, Keskin E, Turan A. Is prostate volume better than PSA density and free/total PSA ratio in predicting prostate cancer in patients with PSA $2.5-10 \mathrm{ng} / \mathrm{mL}$ and $10.1-30 \mathrm{ng} / \mathrm{mL}$ ? Aging Male. 2020;23:59-65. 
9. Zlotta AR, Djavan B, Marberger M, Schulman CC. Prostate specific antigen density of the transition zone: a new effective parameter for prostate cancer prediction. J Urol. 1997;157:1315-21.

10. D'Amico AV, Whittington R, Malkowicz SB, Schultz D, Blank K, Broderick GA, et al. Biochemical outcome after radical prostatectomy, external beam radiation therapy, or interstitial radiation therapy for clinically localized prostate cancer. JAMA. 1998;280:969-74.

11. Kalish J, Cooner WH, Graham SD Jr. Serum PSA adjusted for volume of transition zone (PSAT) is more accurate than PSA adjusted for total gland volume (PSAD) in detecting adenocarcinoma of the prostate. Urology. 1994;43:601-6.

12. Freedland SJ, Isaacs WB, Platz EA, Terris MK, Aronson WJ, Amling CL, et al. Prostate size and risk of high-grade, advanced prostate cancer and biochemical progression after radical prostatectomy: a search database study. J Clin Oncol. 2005;23:7546-54.

13. Freedland SJ, Wieder JA, Jack GS, Dorey F, deKernion JB, Aronson WJ. Improved risk stratification for biochemical recurrence after radical prostatectomy using a novel risk group system based on prostate specific antigen density and biopsy Gleason score. J Urol. 2002;168:110-5.

14. Alcaraz A, Hammerer P, Tubaro A, Schröder FH, Castro R. Is there evidence of a relationship between benign prostatic hyperplasia and prostate cancer? Findings of a literature review. Eur Urol. 2009;55:86473.

15. Prakash K, Pirozzi G, Elashoff M, Munger W, Waga I, Dhir R, et al. Symptomatic and asymptomatic benign prostatic hyperplasia: molecular differentiation by using microarrays. Proc Natl Acad Sci U S A. 2002;99:7598-603.

16. Hammarsten J, Högstedt B. Hyperinsulinaemia: a prospective risk factor for lethal clinical prostate cancer. Eur J Cancer. 2005;41:2887-95.

17. Irani J, Lefebvre O, Murat F, Dahmani L, Doré B. Obesity in relation to prostate cancer risk: comparison with a population having benign prostatic hyperplasia. BJU Int. 2003;91:482-4.

18. Nandeesha H, Koner BC, Dorairajan LN, Sen SK. Hyperinsulinemia and dyslipidemia in non-diabetic benign prostatic hyperplasia. Clin Chim Acta. 2006;370:89-93.

19. Wolff JM, Scholz A, Boeckmann W, Jakse G. Differentiation of benign prostatic hyperplasia and prostate cancer employing prostatic-specific antigen density. Eur Urol. 1994;25:295-8; discussion 304.

20. Elliott CS, Shinghal R, Presti JC. The performance of prostate specific antigen, prostate specific antigen density and transition zone density in the era of extended biopsy schemes. J Urol. 2008;179:1756-61; discussion 1761. 
21. Lee J, Yang SW, Jin L, Lee CL, Lee JY, Shin JH, et al. Is PSA density of the peripheral zone as a useful predictor for prostate cancer in patients with gray zone PSA levels? BMC Cancer. 2021;21:472.

22. Verma A, Onge JS, Dhillon K, Chorneyko A. PSA density improves prediction of prostate cancer. Can J Urol. 2014;10.

23. Wang C, Wang YY, Wang SY, Ding JX, Ding M, Ruan Y, et al. Peripheral zone PSA density: a predominant variable to improve prostate cancer detection efficiency in men with PSA higher than $4 \mathrm{ng}$ ml-1. Asian J Androl. 2021;23:415-20.

24. Aksoy Y, Oral A, Aksoy H, Demirel A, Akcay F. PSA density and PSA transition zone density in the diagnosis of prostate cancer in PSA gray zone cases. Ann Clin Lab Sci. 2003;33:320-3.

25. Liu J, Wang ZQ, Li M, Zhou MY, Yu YF, Zhan WW. Establishment of two new predictive models for prostate cancer to determine whether to require prostate biopsy when the PSA level is in the diagnostic gray zone (4-10 ng ml- 1). Asian J Androl. 2020;22:213-6.

26. Loeb S, Catalona WJ. The Prostate Health Index: a new test for the detection of prostate cancer. Ther Adv Urol. 2014;6:74-7.

27. Carroll PH, Mohler JL. NCCN guidelines updates: prostate cancer and prostate cancer early detection. J Natl Compr Canc Netw. 2018;16:620-3.

28. Chiu ST, Cheng YT, Pu YS, Lu YC, Hong JH, Chung SD, et al. Prostate health index density outperforms prostate health index in clinically significant prostate cancer detection. Front Oncol. 2021;11:772182.

29. Richardson TD, Wojno KJ, Liang LW, Giacherio DA, England BG, Henricks WH, et al. Half-life determination of serum free prostate-specific antigen following radical retropubic prostatectomy. Urology. 1996;48:40-4.

30. Aprikian S, Luz M, Brimo F, Scarlata E, Hamel L, Cury FL, et al. Improving ultrasound-based prostate volume estimation. BMC Urol. 2019;19:68.

\section{Figures}


s

\section{Assessment for eligibility}

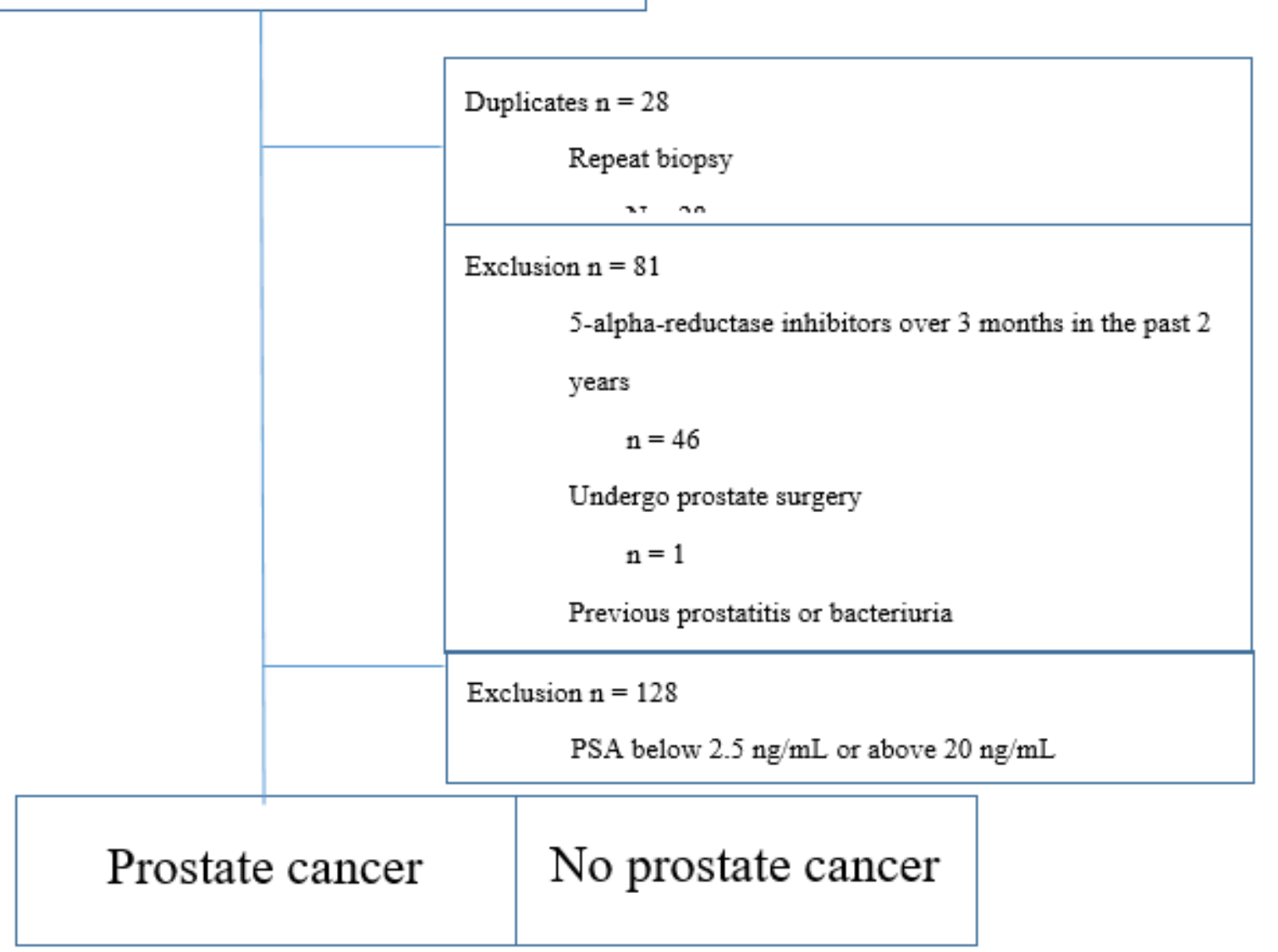

\section{Figure 1}

Flowchart of patient selection

DRE: digital rectal examination; PSA: prostate-specific antigen; ASAP: Atypical Small Acinar Proliferation; PIN: Prostate Intraepithelial Neoplasia 


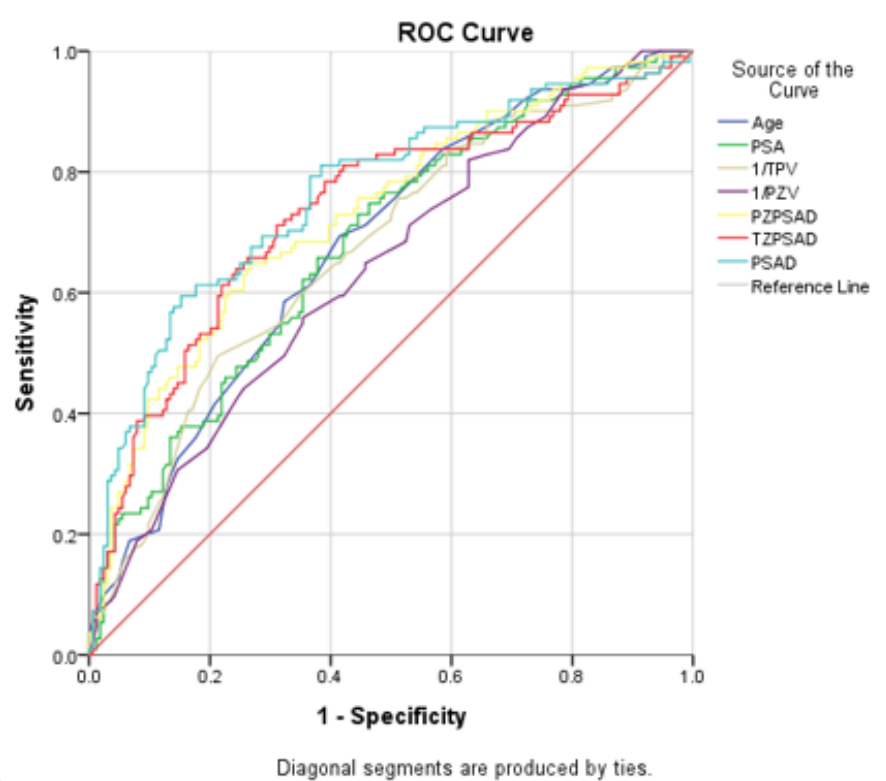

(a)

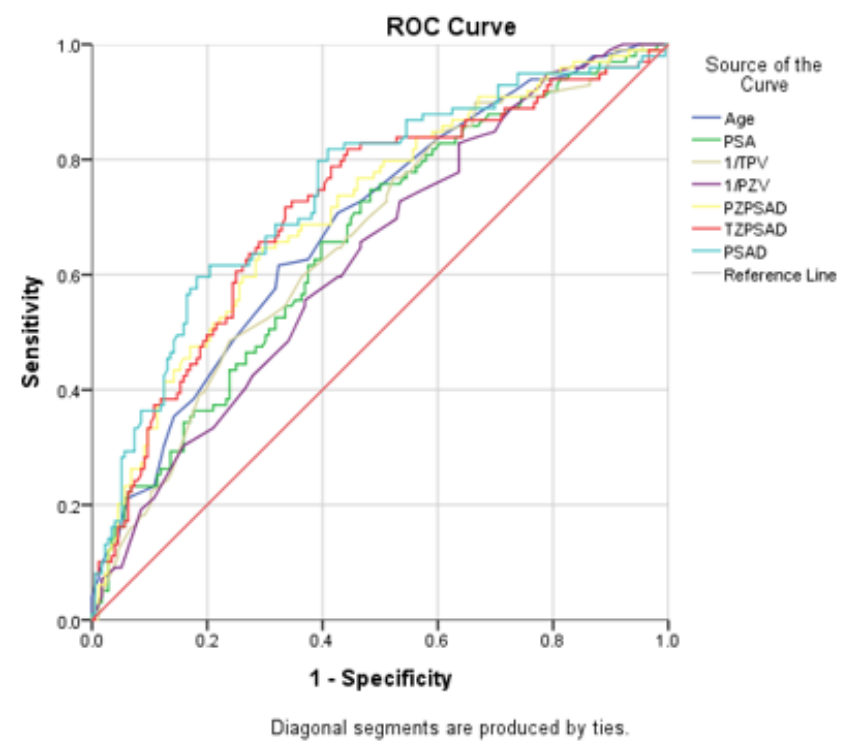

(b)

\section{Figure 2}

Receiver operating characteristic curve analyses.

Receiver operating characteristic curve analyses of age, PSA, TPV, PZV, PZ-PSAD, TZ-PSAD, and PSAD in patients with PSA values of $2.5-20.0 \mathrm{ng} / \mathrm{mL}$ in detecting (a) prostate cancer and (b) clinically significant prostate cancer.

PSA: prostate-specific antigen; TPV: total prostate volume; PZV: peripheral zone volume; PZ-PSAD: peripheral zone prostate-specific antigen density; TZ-PSAD: transition zone prostate-specific antigen density. 


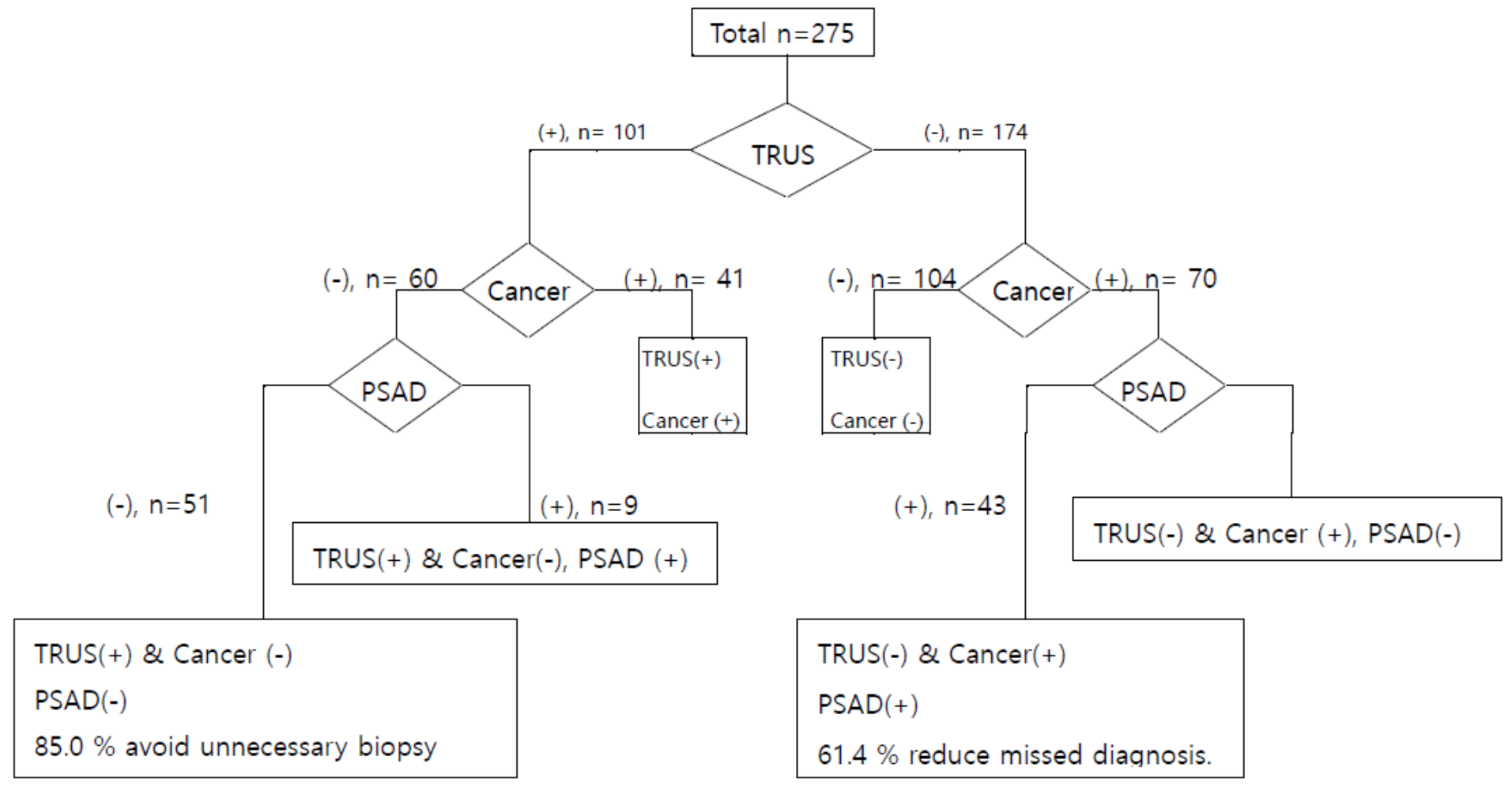

\section{Figure 3}

Cancer diagnosis using TRUS and PSAD in patients with PSA levels of $2.5-20.0 \mathrm{ng} / \mathrm{mL}$

TRUS: transrectal ultrasound; PSAD: prostate-specific antigen density; PSA: prostate-specific antigen; (-): negative; (+): positive; TRUS(+): abnormality findings in TRUS; TRUS(-): no abnormality findings in TRUS; $\operatorname{PSAD}(-)$ : $P S A D<0.218 ; \operatorname{PSAD}(+)$ : PSAD $\geq 0.218$. 